\title{
Efecto de la temperatura sobre la bioacumulación de hidrocarburos en el Ostión americano Crassostrea virginica
}

\section{Temperature effect on bioaccumulation of hydrocarbons in american oysters Crassostrea virginica}

\author{
María Luisa Salas-Salazar, Pablo San Martin-Del Angel, Rodrigo Cuervo-González, Catya Jiménez-Torres, \\ Rosa Idalia Hernández-Herrera*
}

Laboratorio de Biotecnología Ambiental Universidad Veracruzana, Facultad de Ciencias Biológicas y Agropecuarias. Calle Dr. Gabriel Garzón Cossta s/n Col. Universitaria, CP. 92860. Tuxpan, Veracruz, México.

${ }^{*}$ Autor de correspondencia: idhernandez @uv.mx

Artículo científico recibido: 20 de enero de 2016, aceptado: 19 de agosto de 2016

RESUMEN. Diversos estudios muestran el efecto de la temperatura en la bioacumulación de contaminantes en moluscos bivalvos, pero la influencia de esté factor en la bioacumulación de hidrocarburos (HC) en Crassostrea virginica no se ha reportado. En este trabajo se estudió el efecto de la temperatura sobre la acumulación de $\mathrm{HC}$ en $C$. virginica en condiciones controladas, la tasa de sobrevivencia (TS) y el desarrollo gametogénico. Se aplicaron cuatro tratamientos con diferentes temperaturas y concentración de $\mathrm{HC}$ : A ( sin $\mathrm{HC}$ a $\left.26{ }^{\circ} \mathrm{C}\right), \mathrm{B}\left(\sin \mathrm{HC}\right.$ a $\left.30{ }^{\circ} \mathrm{C}\right), \mathrm{C}\left(\operatorname{con~} \mathrm{HC}\right.$ a $\left.26{ }^{\circ} \mathrm{C}\right)$ y $\mathrm{D}$ (con $\mathrm{HC}$ a $30^{\circ} \mathrm{C}$ ) y el control (sin $\mathrm{HC}$ a temperatura ambiente). Los resultados mostraron que el control y los tratamientos $\mathrm{A}$ y $\mathrm{B}$ tuvieron los valores más bajos de bioacumulación de $\mathrm{HC}$ con cantidades menores de $30 \mathrm{mg}$ $\mathrm{kg}^{-1}$. En el tratamiento $C$ se estimaron valores de acumulación de $83 \mathrm{mg} \mathrm{kg}^{-1}$. Mientras que en el tratamiento $\mathrm{D}$ se registraron los niveles más altos de HC con $118 \mathrm{mg} \mathrm{kg}^{-1}$ y la TS más baja (53\%). La TS mayor (93\%) se registró en el control y los tratamientos $\mathrm{A}$ y $\mathrm{C}$. A nivel celular no se identificó ninguna patología, pero el desarrollo gonádico fue afectado por la presencia de $\mathrm{HC}$ y la temperatura de $30^{\circ} \mathrm{C}$, con escaso crecimiento gonadal, predominando organismos indefinidos. Las diferentes temperaturas no influyeron en la bioacumulación de $\mathrm{HC}$ en los organismos, pero se observa efecto negativo en el desarrollo gametogénico y la TS.

Palabras clave: Gametogénesis; molusco; petróleo; sedimento; tasa de sobrevivencia

ABSTRACT. Several studies show the effect of temperature on the bioaccumulation of contaminants in bivalve mollusks, but the influence of this factor on the bioaccumulation of hydrocarbons (HC) in Crassostrea virginica has not been reported. In this work the effect of temperature on $\mathrm{HC}$ accumulation in $C$. virginica under controlled conditions, survival rate (SR) and gametogenic development was studied. Four treatments with different temperatures and an $\mathrm{HC}$ concentration were studied: A (without $\mathrm{HC}$ at $26{ }^{\circ} \mathrm{C}$ ), B (with $\mathrm{HC}$ at $30{ }^{\circ} \mathrm{C}$ ), $\mathrm{C}$ (with $\mathrm{HC}$ at $26{ }^{\circ} \mathrm{C}$ ) and $\mathrm{D}$ (with $\mathrm{HC}$ a $30{ }^{\circ} \mathrm{C}$ ) and the control (without $\mathrm{HC}$ at ambient temperature). Results showed that the control and treatments $\mathrm{A}$ and $\mathrm{B}$ had the lowest $\mathrm{HC}$ bioaccumulation values with amounts below $30 \mathrm{mg} \mathrm{kg}^{-1}$. In treatment $\mathrm{C}$, accumulation values of $83 \mathrm{mg} \mathrm{kg}^{-1}$ were obtained, while treatment $\mathrm{D}$ recorded the highest $\mathrm{HC}$ level, $118 \mathrm{mg} \mathrm{kg}^{-1}$, and the lowest SR (53\%). The highest SR (93\%) was recorded in the control and treatments A and C. At the cellular level no pathology was identified, but gonadal development was affected by the presence of $\mathrm{HC}$ and the temperature of $30{ }^{\circ} \mathrm{C}$, with little gonadal growth and indefinite organisms predominating. The different temperatures did not influence $\mathrm{HC}$ bioaccumulation in the organisms, but a negative effect on gametogenic development and SR was observed.

Key words: Gametogenesis; mollusk; petroleum; sediment; survival rate 


\section{INTRODUCCIÓN}

Los moluscos bivalvos se utilizan como bioindicadores para estimar los tipos y cantidades de contaminantes presentes en ambientes acuáticos, dada su distribución cosmopolita y habilidad de bioacumularlos (Oliver et al. 2001, Bebianno y Berreira 2009, Torres et al. 2012). Existen diversos factores que intervienen en el proceso de bioacumulación, como la biología de la especie de bivalvo, la tasa de filtración, la tasa de absorción, el contenido lipídico y la capacidad de detoxificación, además de factores externos como el punto de muestreo de las fuentes contaminantes, la salinidad, cantidad de materia orgánica y la temperatura ambiental (León et al. 2013).

La acumulación de hidrocarburos $(\mathrm{HC})$ en el tejido lipídico de los organismos, es uno de los principales riesgos para los organismos bioacumuladores (Pereira et al. 1999). Algunas investigaciones demuestran que la exposición de bivalvos a hidrocarburos y metales pesados puede causar daño a nivel celular, en la reproducción y el crecimiento de los organismos (Gold et al. 1995, Oliver et al. 2001). También se ha determinado que la temperatura es un factor que afecta a los organismos ectotermos influyendo en sus reacciones químicas y fisiológicas (Gordon 2005, Ivanina et al. 2008, Sokolova y Lanning 2008). Se ha registrado que la exposición prolongada del bivalvo Crassostrea virginica a altas temperaturas y a metales pesados, afecta los procesos bioquímicos y provoca maduración gonádica temprana (Ruddy et al. 1975). Además, temperaturas elevadas coadyuvan a la acumulación de altas concentraciones de sustancias xenobioticas en los tejidos, asociada a una elevada actividad de hematocitos (Lüchman et al. 2011). Por lo que la temperatura es un factor influyente en el proceso de bioacumulación de $\mathrm{HC}$ y puede relacionarse con afectaciones en el desarrollo gonadal. Por tal motivo el objetivo del estudio fue determinar el efecto de la temperatura en la bioacumulación de hidrocarburos en $C$. virginica, la influencia en la tasa de sobrevivencia y el desarrollo gametogénico.

\section{MATERIALES Y MÉTODOS}

\section{Colecta de sedimento}

Se colectaron $50 \mathrm{~kg}$ de sedimento de la laguna de Tamiahua en la localidad de San Jerónimo, municipio de Tamalín en la zona norte del Estado de Veracruz, México. El área se caracteriza por estar contaminada con hidrocarburos en el ambiente acuático y terrestre. El sedimento se transportó al Laboratorio de Biotecnología Ambiental de la Facultad de Ciencias Biológicas y Agropecuarias de la Universidad Veracruzana, donde se evaluó la concentración de hidrocarburos en el sedimento, mediante análisis de detección y cuantificación, de acuerdo con Fernández et al. (2006).

\section{Colecta de organismos}

Se obtuvieron de la cooperativa pesquera "Del Puerto de Tuxpan" 100 organismos de C. virginica adultos con medidas entre 80 y $87 \mathrm{~mm}$. Los cuales se transportaron al Laboratorio de Biotecnología Ambiental de la Facultad de Ciencias Biológicas y Agropecuarias de la Universidad Veracruzana, donde se realizó el proceso de purga, que consistió en recambios de agua cada 48 h durante $20 \mathrm{~d}$, utilizando agua salobre artificial preparada con agua estéril y sal para acuario. Al finalizar el proceso de purga, se midió la longitud total en $\mathrm{mm}$ de cada organismo con un vernier con precisión de $0.1 \mathrm{~mm}$, y se obtuvo peso en $g$ con una balanza digital con precisión de $0.1 \mathrm{~g}$.

\section{Diseño experimental}

Se utilizaron recipientes de vidrio de $50 \mathrm{~cm}$ de largo, $28 \mathrm{~cm}$ de ancho y $30 \mathrm{~cm}$ de altura, con capacidad de $50 \mathrm{~L}$. Para regular la temperatura se utilizaron termostatos y motor aireador para acuario, manteniendo los parámetros promedio de oxígeno disuelto (OD) de $7.8 \mathrm{mg} \mathrm{L}^{-1}$ y $30 \mathrm{ppm}$ de salinidad. En cada recipiente se colocaron 15 organismos de C. virginica por $30 \mathrm{~d}$. Con base al promedio anual de temperatura superficial del agua en la laguna de Tamiahua de 26 a $30^{\circ} \mathrm{C}$, se evaluaron cuatro tratamientos. Los cuales fueron tratamiento $\mathrm{A}$ ( $\sin$ $\mathrm{HC}$ a $\left.26{ }^{\circ} \mathrm{C}\right), \mathrm{B}\left(\sin \mathrm{HC}\right.$ a $\left.30^{\circ} \mathrm{C}\right), \mathrm{C}\left(\mathrm{HC}\right.$ a $\left.26^{\circ} \mathrm{C}\right)$, 
D $\left(\mathrm{HC}\right.$ a $\left.30^{\circ} \mathrm{C}\right)$ y control (sin $\mathrm{HC}$ a temperatura ambiente).

\section{Extracción y cuantificación de HC en sedi-} mento

Para determinar la cantidad de $\mathrm{HC}$ totales en el sedimento utilizado en los tratamientos, se realizó la extracción y cuantificación del contaminante de acuerdo con la técnica del método de reflujo con el equipo Soxhlet, tomando como referencia los métodos de la Sociedad Estadounidense para Pruebas y Materiales ASTM D5369-93 (ASTM 2005) y de la Agencia de Protección Ambiental de Estados Unidos, EPA 3540 C y 3541 (EPA 1994, EPA 1996).

\section{Extracción y cuantificación de HC en Organis- mos}

Se realizó la extracción en cada uno de los organismos al término de la exposición. Previamente se llevó a cabo la disección para identificar y extraer las gónadas. Las cuales se fijaron en Buffer Fosfato Salino con paraformaldehido para histología al $4 \%$ ( $\mathrm{pH} \mathrm{7.2)} \mathrm{hasta} \mathrm{su} \mathrm{utilización.} \mathrm{El} \mathrm{resto} \mathrm{del}$ organismo se consideró como tejido blando, el cual se empleo para extraer el hidrocarburo acumulado. El tejido se secó en una estufa FELISA a $35{ }^{\circ} \mathrm{C}$ por $24 \mathrm{~h}$, con la finalidad de eliminar la humedad y obtener el peso neto de cada organismo. Luego se maceró hasta lograr una mezcla homogénea, para realizar la extracción con el método de reflujo en un equipo Soxhlet. La cuantificación de HC se realizó calculando el porcentaje de extracto obtenido con la fórmula: \% $E E=\left(\frac{P 2-P 1}{P m}\right) 100$, donde: $E E=$ extracto etéreo, $P 2=$ peso del matraz más extracto obtenido, $P 1=$ peso constante del matraz vacío y $P m=\mathrm{g}$ de muestra (García 2005).

\section{Parámetros biológicos}

Se determinó la tasa de sobrevivencia de los organismos utilizando la fórmula: $T S=$ $\left(\frac{\text { No. Final de organismos }}{\text { No. Inicial de organismos }}\right) 100$, donde $T S=$ tasa de sobrevivencia, la cual se obtuvo a los 15 y $30 \mathrm{~d}$ de exposición a los tratamientos.

\section{Análisis histológico}

Se llevó a cabo al finalizar el experimento para lo cual se utilizaron las gónadas fijadas en la disección. Las muestras se bañaron en sacarosa en concentraciones de $10 \%, 20 \%$ y $30 \%$ a $4{ }^{\circ} \mathrm{C}$ por $24 \mathrm{~h}$, o hasta que las muestras descendieron al fondo de los frascos contenedores. Terminado el proceso del baño en sacarosa, se llevó a cabo la inclusión en resina (NEG 50) en microtubos de plástico, manteniéndolos por $24 \mathrm{~h}$ a temperatura ambiente. Transcurrido el tiempo se efectuó el proceso de corte utilizando un micrótomo de congelación Microm a $25{ }^{\circ} \mathrm{C}$, realizando cortes transversales de las gónadas con un grosor de $7 \mu \mathrm{m}$, los cuales se colocaron en porta objetos esmerilados bañados en poli-L-lisina al $1 \%$. Para mejorar la apariciencia de la estructura celular se realizó la tinción de los cortes con la técnica de Hematoxilina-Eosina. Los cortes obtenidos se analizaron con ayuda de un microscopio compuesto, mediante el cual se identificó la presencia de gametos sexuales masculinos (espermatogonias) o femeninos (ovocitos) y se determinó la etapa del estadio gametogénico.

\section{Análisis Estadísticos}

Los datos se analizaron con el programa estadístico STATISTICA 7 y GraphPad Prism 6 para Windows, realizando un análisis de varianza de una vía y análisis de comparación múltiple de medias de Tuckey a $\mathrm{p}<0.05$.

\section{RESULTADOS}

\section{Contenido de hidrocarburo}

La concentración de hidrocarburo en el sedimento fue de $929.29 \mathrm{mg} \mathrm{kg}^{-1}$ de HC, lo que se clasifica como fracción ligera de acuerdo con la Norma Mexicana NOM-138-SEMARNAT/SS-2012. En cuanto al contenido de $\mathrm{HC}$ en los organismos el valor más bajo se observó en el tratamiento control con $18 \pm 2 \mathrm{mg} \mathrm{kg}^{-1}$ de $\mathrm{HC}$ en el $20 \%$ de organismos. Mientras que en los tratamientos $\mathrm{A}$ y $\mathrm{B}$ se estimaron concentraciones de $25 \pm 4.08$ y $29 \pm 1.83 \mathrm{mg} \mathrm{kg}^{-1}$ de $\mathrm{HC}$, respectivamente. En tanto que en el tratamiento $C$ los organismos pre- 


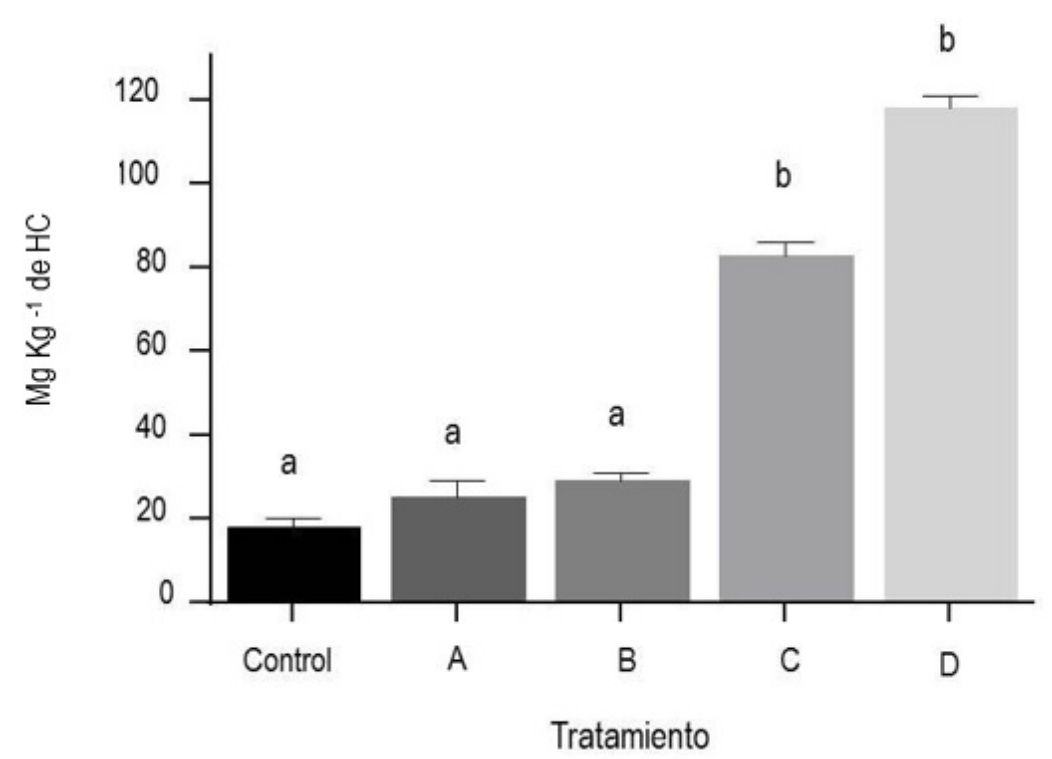

Figura 1. Contenido de hidrocarburos en tejido blando del control y los tratamientos. Letras diferentes en columnas muestran diferencias significativas.

sentaron un mayor contenido de $\mathrm{HC}$ con $83 \pm 3 \mathrm{mg}$ $\mathrm{kg}^{-1}$. Encontrándose la mayor bioacumulación en el tratamiento $D$ con $118 \pm 3 \mathrm{mg} \mathrm{kg}{ }^{-1}$. No se encontraron diferencias significativas en el contenido de $\mathrm{HC}$ entre el control y los tratamientos sin $\mathrm{HC}$ $(A, B)$, mientras que en los tratamientos expuestos a $\mathrm{HC}(\mathrm{C}, \mathrm{D})$ el contenido de estos compuestos fue significativamente mayor $(p<0.05)$ (Figura 1$)$.

\section{Parámetros biológicos}

Los organismos tuvieron una talla promedio de $80 \pm 0.3 \mathrm{~mm}$ por lo que se consideran adultos. La tasa de sobrevivencia (TS) determinó que el control y los tratamientos $\mathrm{A}, \mathrm{B}$ y $\mathrm{C}$ no mostraron diferencias significativas. Los organismos de estos tratamientos y el control tuvieron el $100 \%$ de sobrevivencia los primeros $15 \mathrm{~d}$. Para el control, y los tratamientos A y $\mathrm{C}$ este porcentaje se redujo al 93 $\%$ a los $30 \mathrm{~d}$, mientras que para el tratamiento $\mathrm{B}$ se obtuvo una TS del $86 \%$. El tratamiento $\mathrm{D}$, tuvo diferencias significativas $(p=0.04)$ con el resto de los tratamientos. Este mismo tratamiento mostro una menor TS durante todo el experimento, a los 15 d tuvo una TS del $73 \%$ y disminuyó al $53 \%$ al final del experimento (Figura 2A, 2B).

\section{Desarrollo gonadal y análisis histológico}

En los organismos del tratamiento control, A y $\mathrm{C}$ se observó el desarrollo de las gónadas sobre el área visceral. Mientras que en los organismos de los tratamientos B y D no se observó desarrollo de gónadas (Figura 3). Los mayores porcentajes de organismos fueron hembras, con porcentajes entre el 64 y $61 \%$ en el control y los tratamientos $A$ y B. Mientras que los organismos machos se encontraron entre el 21 y $28 \%$ en los tratamientos control y $\mathrm{A}$, además de organismos indefinidos con porcentajes entre 7 y $14 \%$. En los tratamientos C y $\mathrm{D}$ predominaron los individuos indefinidos con porcentajes entre el 42 y $75 \%$, con hembras entre 58 y $25 \%$, respectivamente (Figura 4). En el control y el tratamiento A se observó que los organismos identificados como hembras se encontraban en la fase de gametogénesis avanzada, en ellos se apreció la poca cobertura de tejido conjuntivo y el desarrollo de folículos con presencia de ovocitos en formación, adheridos a las paredes foliculares. En algunos casos se observaron agrupaciones de folícu- 
A)

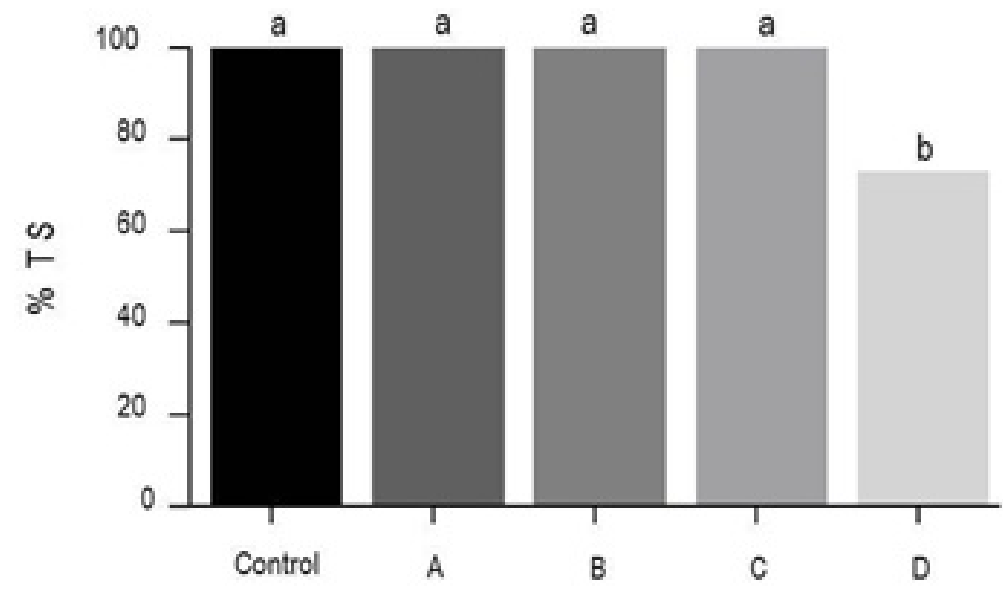

Tratamiento

B)

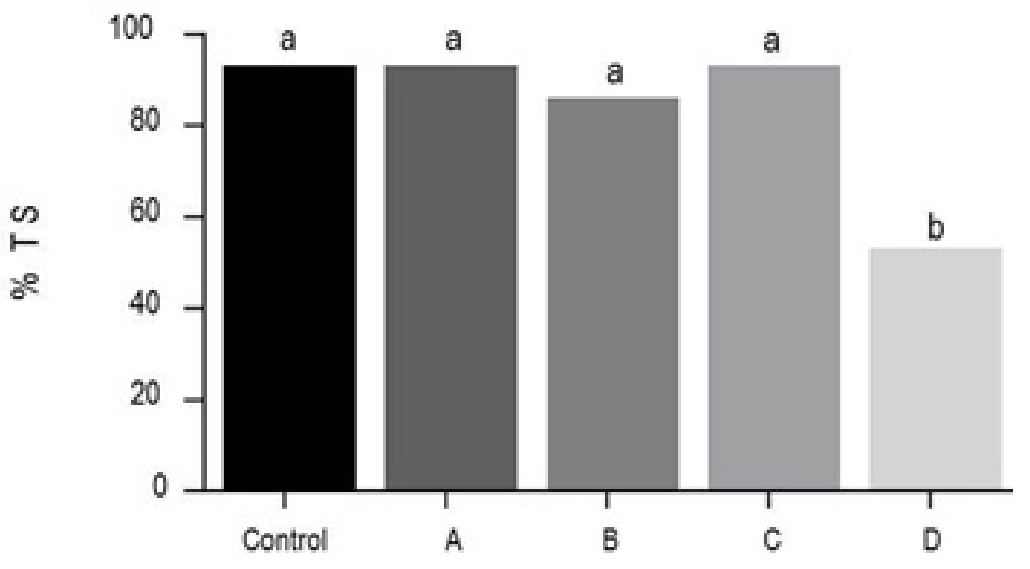

Tratamiento

Figura 2. A) Tasa de sobrevivencia en el control y los tratamientos a los $15 \mathrm{~d}$ de experimentación. B) Tasa de sobrevivencia en el control y los tratamientos a los $30 \mathrm{~d}$ de experimentación. Letras diferentes en columnas muestran diferencias significativas.

los anastomosados donde se distinguieron ovocitos en desarrollo. En el caso de los machos se identificaron dos fases; gametogénesis inicial, en la que se observa la presencia de folículos y espermatogonias; y gametogénesis avanzada; con una reducción del tejido conjuntivo y desarrollo de espermatocitos primarios. En los organismos pertenecientes al tratamiento $B$ se identificó la fase de gametogénesis inicial, en los que se apreció una amplia cobertura de tejido conjuntivo donde se desarrollaron folículos en formación en modo de islotes, que carecían de ovocitos adheridos a las paredes foliculares (Figura 5). En el tratamiento $C$ se observaron organismos indefinidos en la etapa de gametogénesis inicial, en lo que el tejido conjuntivo estuvo cubierto de folículos con presencia de ovocitos inmaduros. En 


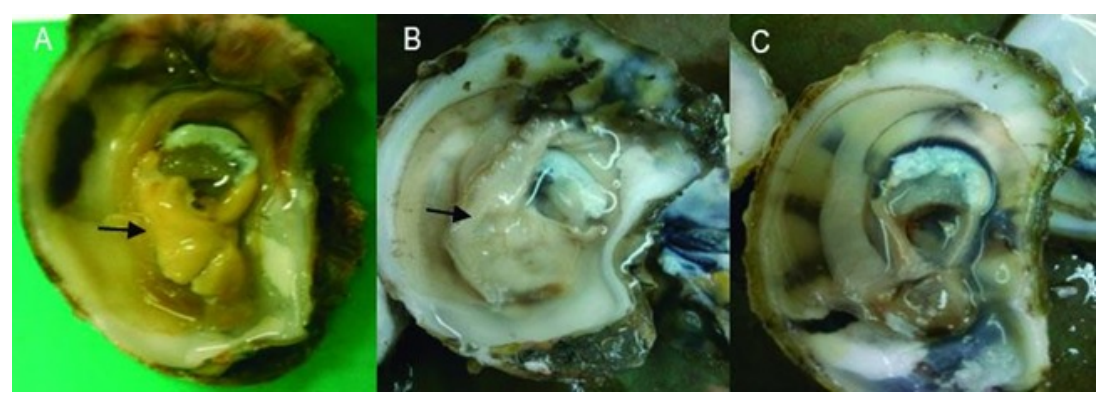

Figura 3. Desarrollo gonadal en los organismos: A) control, B) tratamiento A y C) tratamientos B y D. La flecha señala la gónada.

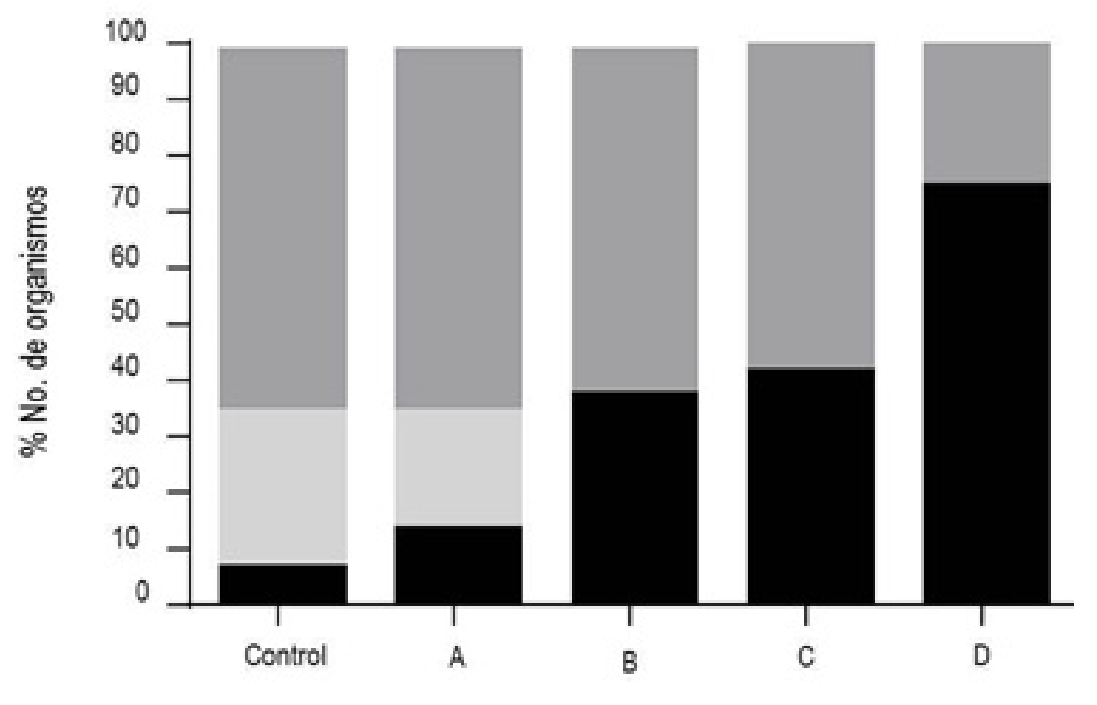

Tratamiento

Figura 4. Porcentajes de sexos identificados en el control y en los diferentes tratamientos. Barra gris oscura corresponde a organismos hembras; barra gris claro a machos y barra negra a organismos indefinidos.

el tratamiento $\mathrm{D}$ los organismos presentaron una cobertura total de tejido conjuntivo con presencia de células mesenquimatosas, por lo que no se logró definir alguna de las etapas de desarrollo gametogénico, no obstante en casos aislados se determinó la etapa de gametogénesis inicial (Figura 6).

\section{DISCUSIÓN}

Los sedimentos contaminados tuvieron una concentración de $929.29 \mathrm{mg} \mathrm{kg}^{-1}$ de HC; valor que es superior a los $440.09 \mathrm{mg} \mathrm{kg}^{-1}$ de HTP en sedimento reportado por San Martin et al. (2012), en la misma área. La diferencia entre ambos valores puede deberse a factores como la topografía y la dirección del flujo del manto freático, además de factores metodológicos como el punto de muestreo seleccionado, lo que indica que hay sitios específicos de mayor contaminación dentro de la misma área. Otros autores han observado que en zonas afectadas por derrames, los niveles de $\mathrm{HC}$ se mantienen altos, aún años después del evento (Tronzynski et al. 2004, Kelly et al. 2008). Las concentraciones promedio de $\mathrm{HC}$ en los individuos no expuestos del control y los tratamientos $A$ y $B$ al final fueron de 


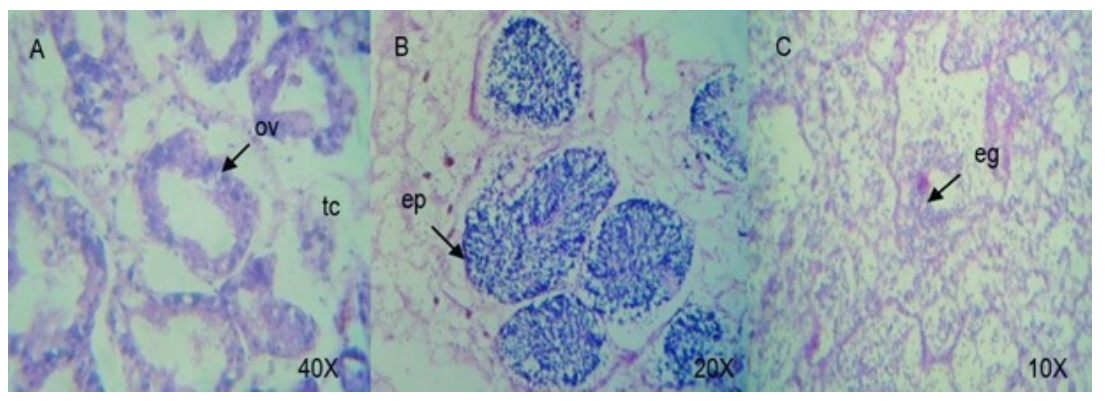

Figura 5. Crassostrea virginica visto al microscopio óptico: A) Hembra del control, gametogénesis avanzada; $B$ ) macho del control, gametogénesis avanzada; C) macho del tratamiento A, gametogénesis inicial. tc tejido conjuntivo; ov ovocito; ep espermatocitos; eg espermatogonias. H-E Hematoxilina-Eosina.

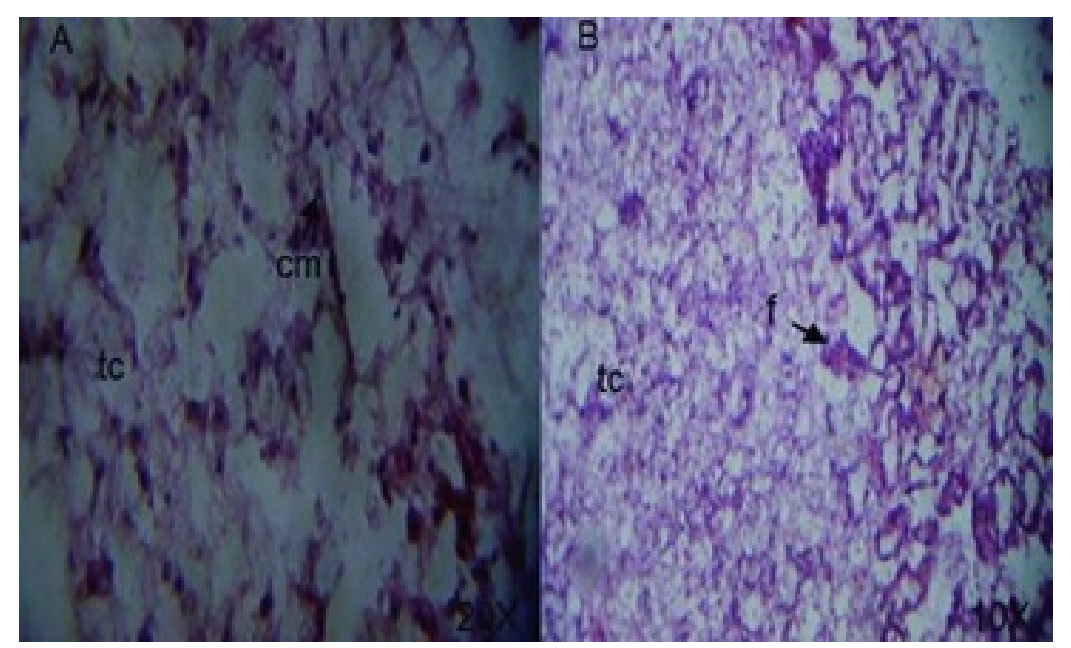

Figura 6. Crassostrea virginica visto al microscopio A) fase indeterminada, tratamiento D. B) Hembra del tratamiento $B$, gametogénesis inicial. tc tejido conjuntivo; $\mathrm{cm}$ células mesenquimatosas; f folículo.

$18 \pm 2,25 \pm 4.08$ y $29 \pm 1.83 \mathrm{mg} \mathrm{kg}^{-1}$ de $\mathrm{HC}$, respectivamente, lo que demuestra la capacidad de los organismos de acumular contaminantes y la baja eliminación del mismo durante la purga. La acumulación de contaminantes en diversas especies de ostras puede darse incluso por poco tiempo de exposición y su permanencia en los organismos puede ser residual aún después de la depuración (Kelly et al. 2008).

La acumulación de $\mathrm{HC}$ en los individuos fue similar a los 26 y $30{ }^{\circ} \mathrm{C}$, lo cual es concordante con lo descrito por Torres et al. (2012) quienes no encontraron diferencias significativas en los niveles de acumulación en organismos de $C$. rhizophorae expuestos a concentraciones de hasta $1406.13 \mathrm{mg}$ $\mathrm{kg}^{-1}$ de $\mathrm{HC}$ en dos temporadas del año. Con valores de bioacumulación de $584.33 \mathrm{mg} \mathrm{kg} \mathrm{kg}^{-1}$, valor que es superior a los $118 \pm 3 \mathrm{mg} \mathrm{kg}{ }^{-1}$ encontrados en el presente estudio. Estas diferencias se pueden atribuir al grado de contaminación y la biodisponibilidad de los HC (Blasco et al. 1999, Blasco et al. 2003). Pero factores biológicos como la talla y el peso, las diferencias fenotípicas y condiciones fisiológicas y reproductivas pueden estar relacionadas en el monitoreo de la contaminación marina (González-Fernández et al. 2016). La TS y la temperatura son factores importantes, presentándose la mayor mortalidad de organismos a los 30 ${ }^{\circ} \mathrm{C}$. Mientras que los organismos del control y de los tratamientos no expuestos a $\mathrm{HC}$ a $26{ }^{\circ} \mathrm{C}$, tuvieron 
TS del $93 \%$, en tanto que los tratamientos no expuestos a $\mathrm{HC}$ a $30{ }^{\circ} \mathrm{C}$ disminuyeron la TS al $86 \%$. En los organismos expuestos a HC, se observó que la TS no fue afectada en los tratamientos a $26^{\circ} \mathrm{C}$, los cuales tuvieron la misma TS que los individuos del control y los del tratamiento sin HC. Se ha determinado que la exposición a contaminantes a temperaturas menores de $26{ }^{\circ} \mathrm{C}$ se puede mantener sobrevivencias del $100 \%$ (Diniz et al. 2008). La TS fue influenciada de forma negativa por la presencia de $\mathrm{HC}$ a $30{ }^{\circ} \mathrm{C}$, observándose la declinación de la misma desde los primeros $15 \mathrm{~d}$ del tratamiento. La reducción observada en la TS se puede atribuir a la sinergia entre el contaminante y la temperatura. Al respecto se sabe que la exposición a metales de C. virginica bajo estrés de temperatura se refleja en alta mortalidad (Lanning et al. 2006).

La maduración gonadal de los organismos, fue afectada en los individuos de los tratamientos B, C y $D$, con efectos mayores en los organismos de los tratamientos $\mathrm{C}$ y $\mathrm{D}$, lo que ocasionó un efecto negativo en el desarrollo de gametos sexuales, con daños mayores en los organismos expuestos a $30^{\circ} \mathrm{C}$, lo que ocasionó el poco o nulo desarrollo de las gónadas, y generó en su mayoría organismos indefinidos. Mientras que en el control y el tratamiento A, se observó el crecimiento de las gónadas. Las afectaciones en el desarrollo gonadal de organismos bivalvos se relacionan con la exposición a fuentes de contaminación, como los hidrocarburos (Cuevas et al. 2015). También los individuos del tratamiento $B$, no expuestos a HC mostraron daños en el desarrollo gonádico. Lo que se atribuye al efecto de la temperatura, debido a que es necesaria una temperatura favorable para el inicio y desarrollo normal de la gametogénesis (Polanco et al. 2002). Al respecto Cuevas et al. (2015) han observado que la severidad de respuesta a la exposición de un agente químico por parte de las ostras depende del grado de exposición al contaminante.

Al analizar de forma histológica las gónadas, se observó que la exposición al tratamiento $D$ no generó ningún tipo de célula anormal o histopatología a nivel gonadal. Al respecto Gold et al. (1995) reportan daños histopatológicos en bran- quias, tracto digestivo, divertículo digestivo y tejido conectivo en organismos de $C$. virginica provenientes de lagunas contaminadas con $\mathrm{HC}$ y metales pesados. Las fases de gametogénesis fueron establecidas en todos los tratamientos y el control. En específico, para el control y el tratamiento A, la fase de gametogénesis avanzada se observó en organismos hembras apreciándose el desarrollo de ovocitos maduros dentro de los folículos. En el caso de organismos machos las fases inicial y avanzada se detectaron y se observó espermatogonias desarrolladas. En los tratamientos $\mathrm{B}$ y $\mathrm{C}$ los organismos se encontraban en la fase de gametogénesis inicial. Mientras que en los tratamientos $C$ y D los organismos se clasificaron como indefinidos, caracterizados por una amplia o total cobertura de tejido conectivo sin desarrollo de gametos. El escaso desarrollo gametogénico observado se puede atribuir a la posible transformación de productos tóxicos derivados de la exposición a las altas temperaturas (Bertilsson y Widenfalk 2002). Al respecto algunos autores señalan que la temperatura y la presencia de metales pesados en el medio, afectan de forma negativa las reacciones fisiológicas de los bivalvos (Gordon 2005, Sokolova y Lanning 2008).

\section{CONCLUSIONES}

La temperatura no es un factor que favorezca la bioacumulación de $\mathrm{HC}$ en $C$. virginica. No obstante, la combinación de HC y elevadas temperaturas afectan de forma negativa la TS, el desarrollo gametogénico de los organismos y la presencia de organismos indefinidos. Estos resultados dan un panorama de los posibles daños ocasionados ante el aumento de la temperatura y la exposición a los hidrocarburos, lo cual podría afectar la producción.

\section{AGRADECIMIENTOS}

Al Consejo Nacional de Ciencia y Tecnología (CONACYT) por otorgar el financiamiento para los estudios de posgrado de la MC. Luisa Salas Salazar. 


\section{LITERATURA CITADA}

ASTM (2005) Annual Book of ASTM Standards. Extraction of solid waste samples for chemical analysis using Soxhlet extraction, D5369. American Society for Testing and Materials. USA. pp: 196-201.

Bebianno M, Barreira L (2009) Polycyclic aromatic hydrocarbons a concentration and biomarkers response in the calm Rudi tapes decussates transplanted in the Ria Formosa lagoon. Ecotoxicology and Environmental Safety 72: 1849-1860.

Bertilsson S, Widenfalk A (2002) Photochemical degradation of PAHs in fresh water and their impact on bacterial growth-influence of water chemistry. Hidrobiologia 469: 23-32.

Blasco J, Arias AM, Sáenz V (1999) Heavy metals in organisms of the river Guadalquivir estuary: possible incidence of the Aznalcóllar disaster. Science of the Total Environment 242: 249-259.

Blasco J, Sáens V, Arias AM, González R, Campana O, Ransome T, et al (2003) Efecto del accidente minero sobre los organismos residentes en el estuario Guadalquivir y en las zonas de la plataforma próxima a la desembocadura, En: Forja J, Gómez A (ed). Contaminación por metales pesados del estuario del Guadalquivir. Servicio publicaciones Universidad de Cádiz, España. pp: 165-203.

Cuevas N, Zorita I, Costa P, Franco J, Larreta J (2015) Development of histopathological indices in the digestive gland and gonad of mussels: Integration with contamination levels and effects of confounding factors. Aquatic Toxicology 162: 152-164.

Diniz MS, Santos HM, Costa PM, Peres I, Costa MH, Alves S, et al. (2008) Effects of exposure to arsenic in Corbicula fluminea: Evaluation of the histological, histochemical and biochemical responses. Ciencias Marinas 34: 307-316.

EPA (1994) Automated soxhlet extraction. SW-846 Test methods for evaluating solid waste physical/chemical methods. 1-10.

EPA (1996) Method 3540C. Soxhlet extraction, SW-846 Manual. Washington, DC: Government Printing Office. 1-8.

Fernández L, Rojas N, Roldán T, Ramírez M, Zegarra H, Hernández R, et al. (2006) Manual de técnicas de análisis de suelos aplicadas a la remediación de sitios contaminados. Secretaría de Medio Ambiente y Recursos Naturales, Instituto Nacional de Ecología. 180p.

García D (2005) Manual de análisis de alimentos. Facultad de ciencias biológicas. Universidad Autónoma de Nuevo León. México. 69p.

Gold G, Sima R, Zapata O, Güemez J (1995) Histopathological effects of petroleum hydrocarbons and heavy metals on the American Oyster (Crassostrea virginica) form Tabasco, Mexico. Marine Pollution Bulletin 31: 439-445.

González-Fernández C, Albentosa M, Campillo J, Viñas L, Franco A, Bellas J (2016) Effect of mussel reproductive status on biomarker responses to PAHs: Implications for large-scale monitoring programs. Aquatic Toxicology 177: 380-394.

Gordon CJ (2005) Temperature and toxicology: An integrative, comparative and environmental approach. Boca Raton, London, New York, Singapore. 329p.

Ivanina AV, Taylor C, Sokolova IM (2008) Effects of elevated temperature and Cadmium exposure on stress protein response in eastern oysters Crassostrea virginica (Gmelin, 1971). Aquatic toxicology 91: 245254. 
Kelly C, Santillo D, Johnston P, Fayad G, Baker KL, Law RJ (2008) Polycyclic aromatic hydrocarbons in oysters from coastal waters of the Lebanon 10 months after the Jiyeh oil spill in 2006. Marine Pollution Bulletin 56: 1215-1233.

Lannig G, Flores J, Sokolova I (2006) Temperature-dependent stress response in oysters, Crassostrea virginica: Pollution reduces temperature tolerance in oysters. Aquatic Toxicology 79: 278?287.

León VM, Moreno-González R, González E, Martínez F, García V, Campillo J (2013) Interspecific comparison of polycyclic aromatic hydrocarbons and persistent organochlorines bioaccumulation in bivalves from a Mediterranean coastal lagoon. Science of the Total Environment 463-464: 975-987.

Lüchman K, Mattos J, Siebert M, Granucci N, Dorrington T, Bicego M, et al. (2011) Biochemical biomarkers and hydrocarbons concentrations in the mangrove oyster Crassostrea brasilliana following exposure to diesel fuel water-accommodated fraction. Aquatic toxicology 105: 652-660.

Oliver ML, Fisher SW, Winstead TJ, Hemmer LB, Long RE (2001) Relationship between tissue contaminants and defense-related characteristics of oysters (Crassostrea virginica) from five Florida bays. Aquatic Toxicology 55: 203-222.

Pereira WE, Hostettler FD, Luoma SN, Van Green A, Fuller CC, Anima RJ (1999) Sedimentary record of anthropogenic and biogenic polycyclic aromatic hydrocarbons in San Francisco bay, California. Marine Chemistry 64: 99-113.

Polanco TE, Fernández PJ, Ruesga BS (2002) Impulso, desarrollo y potenciación de la ostricultura en España. Fundación Alfonso Martínez Escudero. España. 350p.

Ruddy GM, Feng SY, Campbell S (1975) The effect of prolonged exposure to elevated temperatures on the biochemical constituents, gonadal developed and shell deposition of the American oyster, Crassostrea virginica. Comparative Biochemistry Physiology 51: 157-164.

San Martin P, Salas ML, Zarza EA, Hernández RI (2012) Biorremediación de sedimentos contaminados de la laguna de Tamiahua, Veracruz, (Una propuesta de Biorremediación). Academia Mexicana Multidisciplinaria 2: 1-5.

Sokolova IM, Lanning G (2008) Interactive effects of metal pollution and temperature on metabolism in aquatic ectotherms: Implications of global change. Climate Research 37: 181-201.

Torres RJ, Cesar A, Pereira C, Choueri R, Abessa D, Do Nascimento M, et al (2012) Bioaccumulation of polycyclic aromatic hydrocarbons and mercury in oysters (Crassostrea rhizophorae) from two Brazilian estuarine zones. International Journal of Oceanography 2012: 1-8.

Tronczynski J, Munschy C, Héas-Moisan K, Nadège G, Truquet I, Olivier N, et al. (2004) Contamination of the Bay of Biscay by polycyclic aromatic hydrocarbons (PAHs) following the T/V "Erika" oil spill. Aquatic Living resources 17: 243-259. 